Subscriber access provided by Caltech Library

\title{
Letter
}

\section{Monolithic Photoelectrochemical Device for Direct Water Splitting with 19\% Efficiency}

Wen-Hui Cheng, Matthias H. Richter, Matthias M. May, Jens Ohlmann, David Lackner, Frank Dimroth, Thomas Hannappel, Harry A Atwater, and Hans-Joachim Lewerenz

ACS Energy Lett., Just Accepted Manuscript • DOI: 10.1021/acsenergylett.8b00920 • Publication Date (Web): 25 Jun 2018

Downloaded from http://pubs.acs.org on June 26, 2018

\section{Just Accepted}

"Just Accepted" manuscripts have been peer-reviewed and accepted for publication. They are posted online prior to technical editing, formatting for publication and author proofing. The American Chemical Society provides "Just Accepted" as a service to the research community to expedite the dissemination of scientific material as soon as possible after acceptance. "Just Accepted" manuscripts appear in full in PDF format accompanied by an HTML abstract. "Just Accepted" manuscripts have been fully peer reviewed, but should not be considered the official version of record. They are citable by the Digital Object Identifier (DOI@). "Just Accepted" is an optional service offered to authors. Therefore, the "Just Accepted" Web site may not include all articles that will be published in the journal. After a manuscript is technically edited and formatted, it will be removed from the "Just Accepted" Web site and published as an ASAP article. Note that technical editing may introduce minor changes to the manuscript text and/or graphics which could affect content, and all legal disclaimers and ethical guidelines that apply to the journal pertain. ACS cannot be held responsible for errors or consequences arising from the use of information contained in these "Just Accepted" manuscripts. 


\title{
Monolithic Photoelectrochemical Device for Direct
}

\section{Water Splitting with 19\% Efficiency}

\author{
Wen-Hui Cheng ${ }^{1,2}$, Matthias H. Richter ${ }^{2,3}$, Matthias M. May ${ }^{4,5,6}$, \\ Jens Ohlmann ${ }^{7}$, David Lackner ${ }^{7}$, Frank Dimroth ${ }^{7}$, \\ Thomas Hannappel $^{6} *$, Harry A. Atwater ${ }^{1,2} *$, Hans-Joachim Lewerenz ${ }^{2,8}$ \\ 1 Department of Applied Physics and Material Science, California Institute of Technology, \\ Pasadena, CA 91125, USA. \\ 2 \\ Joint Center for Artificial Photosynthesis, California Institute of Technology, Pasadena, \\ CA 91125, USA. \\ 3 Division of Chemistry and Chemical Engineering, California Institute of Technology, \\ Pasadena, CA 91125, USA. \\ 4 Department of Chemistry, University of Cambridge, Lensfield Road, CB2 1EW \\ 5 Cambridge, UK \\ Helmholtz-Zentrum Berlin für Materialien und Energie GmbH, Institute for Solar Fuels, \\ 6 Hahn-Meitner-Platz 1, D-14109 Berlin, Germany. \\ Department of Physics, Technische Universität Ilmenau, Gustav-Kirchhoff-Str. 5, D- \\ 98693 Ilmenau, Germany. \\ 7 Fraunhofer Institute for Solar Energy Systems ISE, Heidenhofstraße 2, D-79110 \\ 8 \\ Freiburg, Germany. \\ Division of Engineering and Applied Science, California Institute of Technology, \\ Pasadena, CA 91125, USA.
}

\section{Corresponding Author}

* E-mail: haa@caltech.edu; thomas.hannappel@tu-ilmenau.de; matthias.may@physik.hu$\underline{\text { berlin.de }}$ 


\begin{abstract}
Efficient unassisted solar water splitting, a pathway to storable renewable energy in the form of chemical bonds, requires optimization of a photoelectrochemical device based on photovoltaic tandem heterojunctions. We report a monolithic photocathode device architecture that exhibits significantly reduced surface reflectivity, minimizing parasitic light absorption and reflection losses A tailored multifunctional crystalline titania interphase layer acts as corrosion protection layer with favorable band alignment between the semiconductor conduction band and the energy level for water reduction, facilitating electron transport at the cathode-electrolyte interface. It also provides a favorable substrate for adhesion of high activity Rh catalyst nanoparticles. Under simulated AM 1.5G irradiation, solar-to-hydrogen efficiencies of $19.3 \%$ and $18.5 \%$ are obtained in acidic and neutral electrolytes, respectively. The system reaches a value of 0.85 of the theoretical limit for photoelectrochemical water splitting for the energy gap combination employed in the tandem-junction photoelectrode structure.
\end{abstract}

\title{
TOC GRAPHICS
}

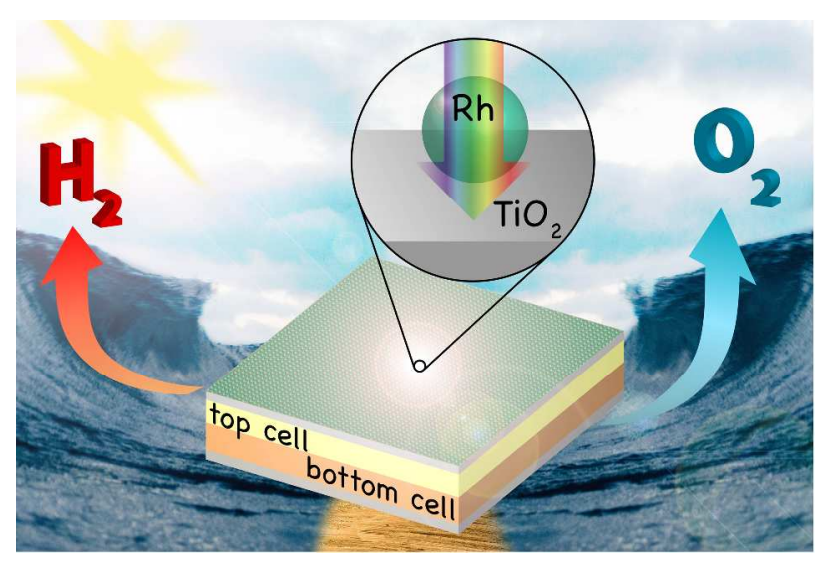


Electrochemical water splitting was achieved by van Trostwijk and Deiman in 1789 and, about a decade later, by Nicholsen and Carlisle ${ }^{1,2}$, whereas light-induced unassisted water splitting with rutile as a photoanode was reported in 1972, resulting in a small, but measurable efficiency ${ }^{3}$. Efficient solar water splitting was first achieved using a dual junction tandem photoelectrode 4 under a light intensity equivalent to 11 suns.

In 2015, several devices with solar-to-hydrogen efficiency (STH) greater than $10 \%$ at 1 sun illumination were reported ${ }^{5}$, and in 2017 an efficiency of $16.2 \%$ was achieved ${ }^{6}$. Overall, advances in solar water splitting ${ }^{1}$ have led to a number of functional prototypes of photoelectrochemical and photoelectrosynthetic cells in recent years ${ }^{2}$, featuring improved photoelectrode stability through the use of corrosion protection layers ${ }^{7,8}$. However, comparison of solar-to-hydrogen efficiencies realized so far with theoretical limiting efficiencies ${ }^{9}$ shows considerable room for further improvement; at present, the highest efficiency systems reach about $2 / 3$ of the theoretical limiting value for a given photoelectrode. To enable solar-tohydrogen efficiencies approaching theoretical limits, the photovoltage has to be as large as possible, which requires a minimized photoelectrode dark current. This in turn dictates that the charge carrier recombination at interfaces must be prevented. To maximize the photocurrent, a reduction of the photoelectrode surface reflectivity under operating conditions is also required, as is mitigation of light absorption in the catalyst layer applied to the photoelectrode surface ${ }^{10}$.

If one utilizes the band gap combination of a given tandem photoelectrode and the best reported exchange current densities for the HER and OER, omits losses due to ERE and solution resistance, the realistic limiting STH efficiencies can be calculated ${ }^{9}$. For the tandem 
photoelectrode used here (see Supporting Information Section S1), this value is $22.8 \%$. Approaching such limiting efficiencies provides a clear objective for a renewable fuels technology, since inclusion of hydrogen in the existing worldwide fuel generation infrastructure could enable direct and widespread application of renewable fuels in the transportation sector and for electricity generation 11 .

Here, we demonstrate an approach to achieving efficiencies near the theoretical limits for the photoelectrode energy bandgaps employed. A key aspect of our approach is (i) the use of a crystalline anatase $\mathrm{TiO}_{2}$ photocathode interfacial layer (see Supporting Information Section S3), deposited by atomic layer deposition (ALD), to facilitate reduced reflectivity and interface recombination velocity, and (ii) a size distribution and spatial arrangement of $\mathrm{Rh}$ catalyst nanoparticles tailored to achieve ultralow light attenuation. The crystalline anatase $\mathrm{TiO}_{2}$ interlayer shows excellent energy band alignment with the tandem window layer and its interfacial ultrathin oxidized surface part and with the electrolyte. In addition, it serves as an efficient antireflection coating and as a support for the catalyst nanoparticles, with enhanced adhesion relative to III-V compound semiconductor surfaces.

We employ a dual-junction tandem photoelectrode where the high band gap subcell thickness has been increased for better current matching and the transparency of the tunnel diode was improved ${ }^{10,12,13}$. To further increase the STH efficiency, interfacial layers have been designed to reduce charge carrier recombination and to increase optical light coupling into the photoelectrode absorber layers. The surface conditioning sequence resulted in etching of the GaAs cap layer by a $\mathrm{NH}_{4} \mathrm{OH}-\mathrm{H}_{2} \mathrm{O}_{2}-\mathrm{H}_{2} \mathrm{O}$ solution, leaving an oxidized surface layer $\left(\mathrm{AlInPO}_{\mathrm{x}}\right)$ on top of the $\mathrm{n}^{+}$-doped AlInP window layer (see Supporting Information Section S1). A 
crystalline anatase $\mathrm{TiO}_{2}$ film with an effective thickness of $30 \mathrm{~nm}$ was deposited to act as a corrosion protection layer and an antireflection coating, as well as serving as conducting substrate surface for photoelectrodeposition of Rh nanoparticle (NP) electrocatalysts. The Rh NPs exhibited large surface areas and thus high exchange current and, simultaneously, particularly low light attenuation. The photocathode device configuration employed is generally less prone to photodecomposition than photoanode devices, where charge carriers with high oxidation potential are present at the semiconductor surface. Fig. 1 shows a schematic of the resulting device: the photoelectrode consisting of GaInP and GaInAs subcells on a GaAs substrate, a anatase $\mathrm{TiO}_{2}$ protective layer, the Rh NPs catalyst layer and a sputtered $\mathrm{RuO}_{2}$ counter electrode (OER) are depicted. Also depicted on the side of the layer structure in Fig. 1 is an energy band diagram under illumination where the quasi Fermi levels show the splitting for electrons and holes necessary to achieve unassisted water splitting (See Supporting Information Section S4). The surface of the crystalline $\mathrm{TiO}_{2}$ film illustrated in Fig. $1 \mathrm{~b}$ indicates a continuous film with height variations, seen by AFM, that give it a flake-like appearance. Fig. 1c illustrates the protocol for pulsed photoelectrodeposition of Rh catalyst nanoparticles (see Supporting Information Section S1) and inset gives SEM and AFM images of the Rh NPs. Fig. 1c depicts the procedure to obtain the highest activity catalysts at almost negligible light attenuation: fine control of particle size smaller than $20 \mathrm{~nm}$ was achieved by careful adjustment of the electrode potential, enabling considerably higher catalyst loading compared to a dense film of equivalent catalyst loading deposited by conventional vapor phase or electrochemical reduction. This procedure facilitates photocathodes with high transparency catalysts, which maintain the high photocurrent densities and result in increased efficiency, which is determined from the relation 


$$
\eta_{S T H}=\frac{J_{o p} \cdot E_{r x n} \cdot f_{F E}}{P_{i n}}
$$

The solar fuel generator efficiency $\eta_{\mathrm{STH}}$ is given by the operating current at the counter electrode potential, the thermodynamic value for the reaction $\left(E_{\mathrm{rxn}}=1.23 \mathrm{~V}\right.$ for water splitting under standard conditions) and by the reaction Faradaic efficiency $f_{\mathrm{FE}}$, determined by gas product analysis measurements.
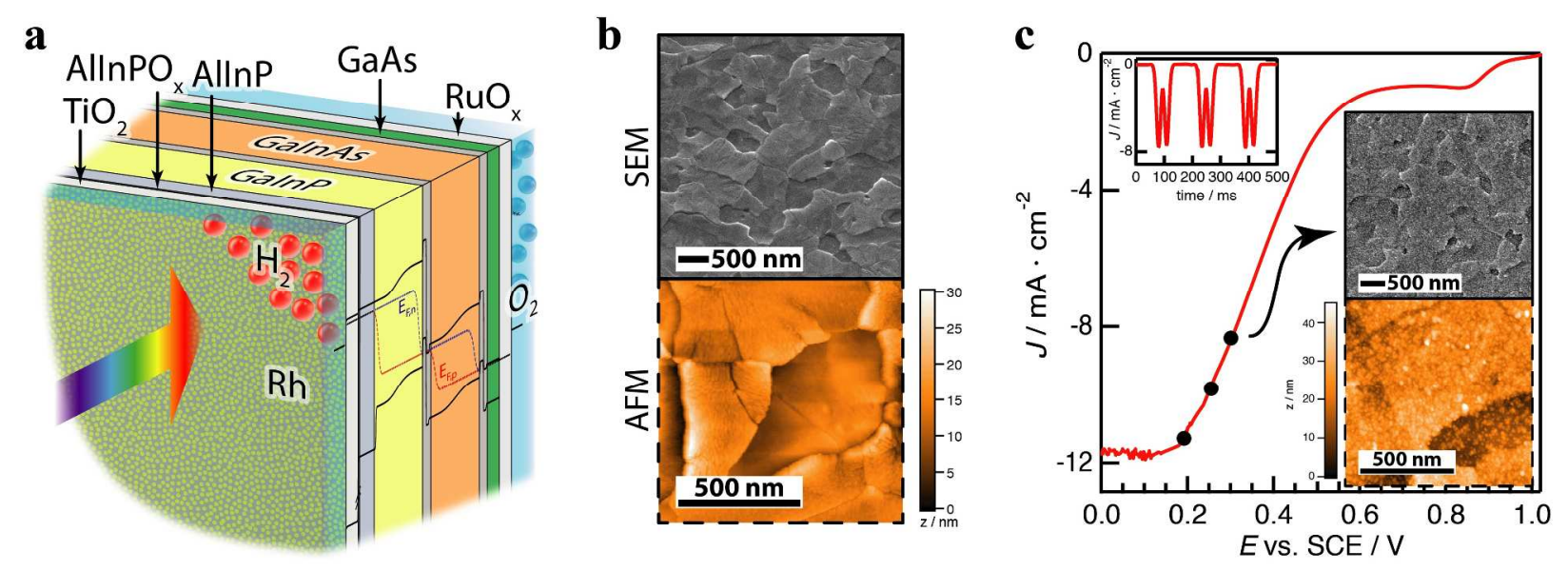

Figure 1. Functionalization of a dual junction tandem as photoelectrode for unassisted water splitting; (a) Schematic of the device structure after functionalization with interfacial films and electrocatalysts (see text); (b) topography of the crystalline anatase $\mathrm{TiO}_{2}$ layer by HRSEM and AFM; (c) protocol of the photoelectrodeposition of Rh NPs; the arrow shows the potential used for stroboscopic deposition under white light illumination as shown in the upper left insert. The potential choices made are indicated by black dots; the best result was obtained for $E=+0.3 \mathrm{~V}$ vs. SCE. Resulting Rh particles are shown in the insert under the J-V characteristic (upper image: SEM, lower one: AFM). The potential control and corresponding particle size distribution 
are included in Supplementary Information Section S1. The root mean square surface roughness of $\mathrm{TiO}_{2}$ is $6.3 \mathrm{~nm}$ and $3.6 \mathrm{~nm}$ (with/without $\mathrm{Rh}$, respectively).

Electronically, the photoelectrode configuration used here facilitates alignment of the conduction bands of the AlInP window layer of the tandem photoelectrode to the indium oxide and indium phosphate layers (created by the cap layer etching process) and the anatase $\mathrm{TiO}_{2}$ protection/antireflective layer. We note that photogenerated electrons, which are minority carriers in the main part of the tandem subcells, become majority carriers in the AlInP and $\mathrm{TiO}_{2}$ layers, reducing recombination losses in carrier transport. Details of the energy band alignment, and the photoelectron spectroscopy and optical data used to support it, are described in the Supporting Information Section S4. In addition, the large valence band offset between AlInP and $\mathrm{TiO}_{2}$ blocks interfacial hole transport, resulting in a small overall reverse saturation current, improving the photovoltage. This feature is important for achieving high STH efficiencies.

The influence of the surface modifications on optical properties and on the photocurrent is shown in Fig. 2. A reduction of the reflectivity by $\sim 15 \%$ is achieved by use of the $\mathrm{TiO}_{2}$ interlayer (Supporting Information Section S5) whereas the Rh NPs in Fig. 2a show negligible additional absorption, which is attributed to the blue-shifted plasmonic resonance of the $\mathrm{Rh}$ nanoparticles. For particle sizes below $20 \mathrm{~nm}$, a shift from the visible region into the ultraviolet one occurs, making the Rh layer almost fully transparent ${ }^{14-16}$. The detailed optimization of the optical design regarding the thickness of $\mathrm{TiO}_{2}$ and $\mathrm{Rh}$ particle size is discussed in Supporting Information Section S6 including both simulations and experiments support. 
The corresponding photocurrent-voltage characteristics in acidic electrolyte demonstrate a pronounced increase in the current and, as expected, also a shift of the bend of the photocurrent characteristic towards more anodic potentials, thereby additionally increasing the photocurrent at the $\mathrm{RuO}_{2}$ counter electrode (OER) potential. The result with incorporation of $\mathrm{TiO}_{2}$ is a relative increase of $28 \%$ of the tandem cell output. An STH efficiency of $19.3 \%$ is obtained at $0 \mathrm{~V}$, with an operating current of $15.7 \mathrm{~mA} / \mathrm{cm}^{2}$, assuming an initial Faradaic efficiency of unity, which is supported by the gas evolution measurements shown in Fig. 3 b. These data represent a $20 \%$ increase in efficiency above the previously reported one sun photoelectrosynthetic cell efficiency benchmark ${ }^{6}$.
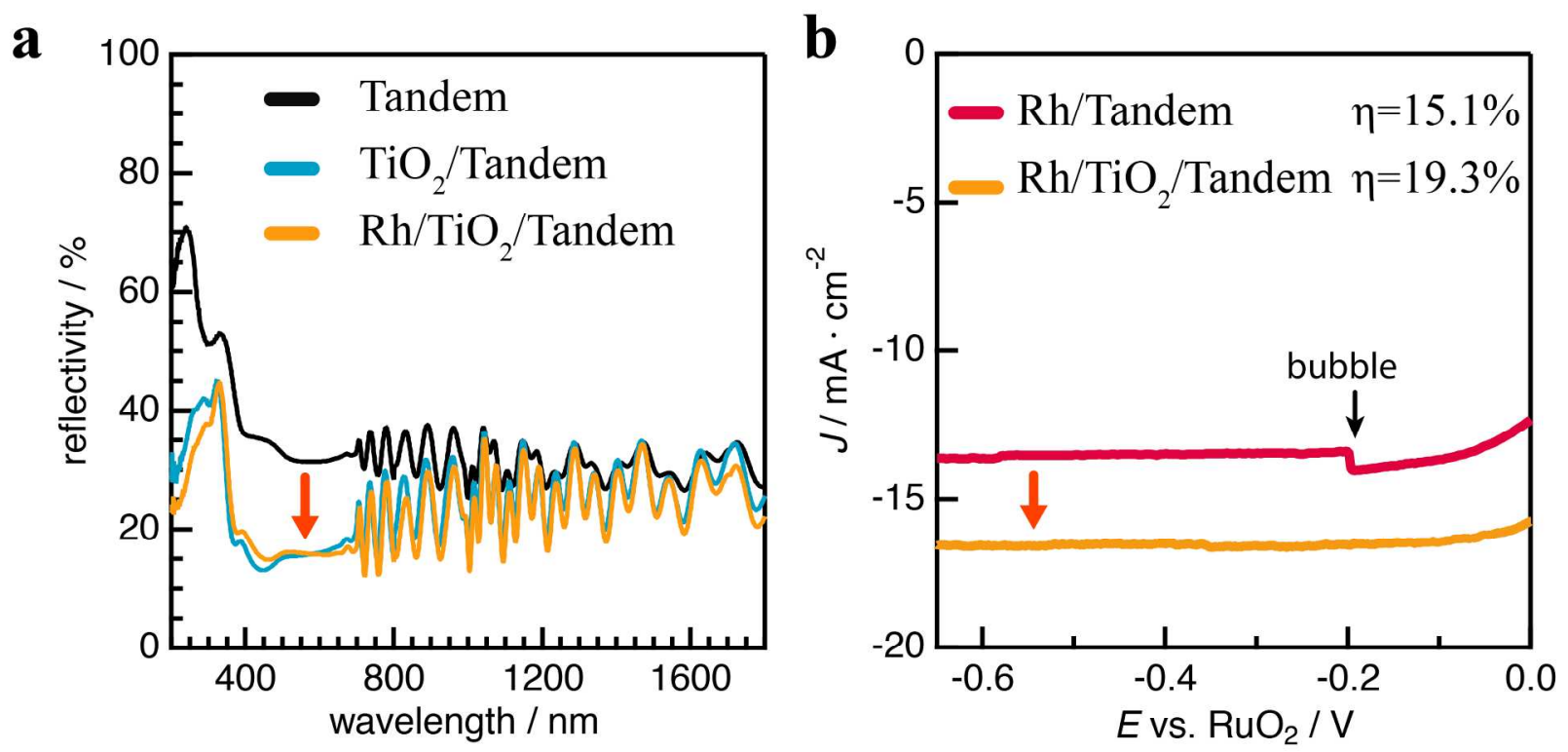

Figure 2. Optoelectronic properties of the surface functionalized electrolyte / $\mathrm{Rh} / \mathrm{TiO}_{2} /$ oxide / AlInP - GaInP / GaInAs / GaAs water splitting device; (a) reflectivity $\mathrm{R}_{\mathrm{a}}$, measured in air, of the dual-junction tandem solar cell without ARC (black curve), secondly reflectivity obtained after 
$\mathrm{TiO}_{2}$ coating (blue curve) and after photoelectrochemically deposited Rh NPs (yellow curve); $\mathrm{R}_{\mathrm{a}}$ is larger than under operation in electrolyte due to the different refractive indices of air and water; (b) comparison of the output characteristics of the tandem device after cap layer etching and of the fully surface functionalized photoelectrode. The orange arrows indicate the improvement after incorporation of the $\mathrm{TiO}_{2}$ layer.

The high photocurrent at $0 \mathrm{~V}$ vs. $\mathrm{RuO}_{2}$ indicates that electron transport is virtually uninhibited from the absorber layer through the indium and phosphorus oxide and $\mathrm{TiO}_{2}$ interfacial layers to the electrolyte. The corresponding energy band relations can be inferred from surface characterization using ultraviolet and X-ray photoelectron spectroscopy. While the simplest approach to assessment of band alignment follows Anderson's idealized model ${ }^{17}$ for planar contacts and does not consider energy band shifts due to surface and/or interface dipoles, this approach certainly does not apply here, as the junctions formed at the AlInP/oxide, oxide/ $\mathrm{TiO}_{2}$ and $\mathrm{TiO}_{2} / \mathrm{Rh} /$ electrolyte interfaces are complex. Thus the energy band diagram of the heterojunction structure was inferred from ultraviolet and X-ray photoelectron spectroscopy measurements, and can be found in the Supporting Information Section S4. It should be noted that equilibrium formation between small metallic catalyst nanoparticles and semiconductors appears to depend on the substrate doping level ${ }^{5}$ and does obviously not follow a Schottky thermionic emission model, in particular in contact with an electrolyte ${ }^{18,19}$. In addition, metal work functions depend on NP size ${ }^{20}$, so comparison of the energy levels of NP catalyst layers 
with planar thin films is notably challenging; therefore, only an estimate of the NP catalyst layer energy level can be given, supported by the device operating data.

The output data shown in Fig. $2 \mathrm{~b}$ were obtained in an acidic electrolyte of $\mathrm{pH} 0$. Figure 3, summarizes the main performance characteristics. Fig. 3a illustrates the photocurrent-voltage characteristics under three conditions: i) at $\mathrm{pH} 0$ with $19.3 \% \mathrm{STH}$, ii) at neutral $\mathrm{pH}$ with $18.5 \%$ STH, and iii) using an anion exchange membrane (AEM) with an STH of $14.8 \%$. The observed unassisted water splitting efficiencies critically depend on the experimental conditions (details about the efficiency benchmarking of our PEC device under AM $1.5 \mathrm{G}$ conditions, as well as a discussion of efficiency accuracy and polarization loss, are given in the Supplementary information section S7).

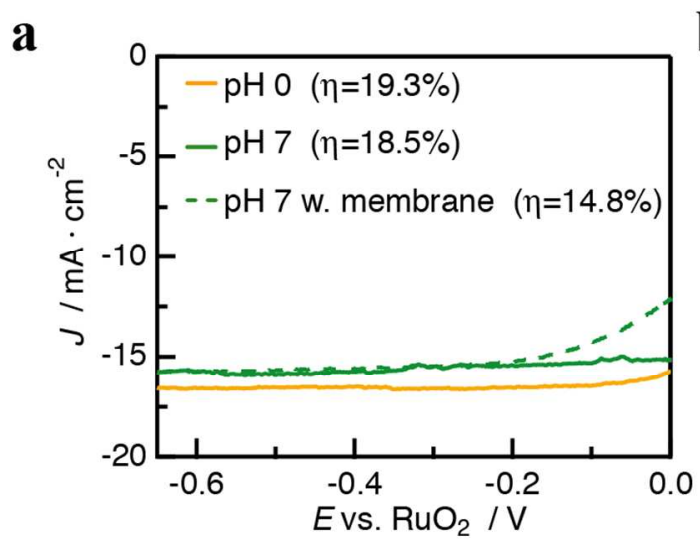

b

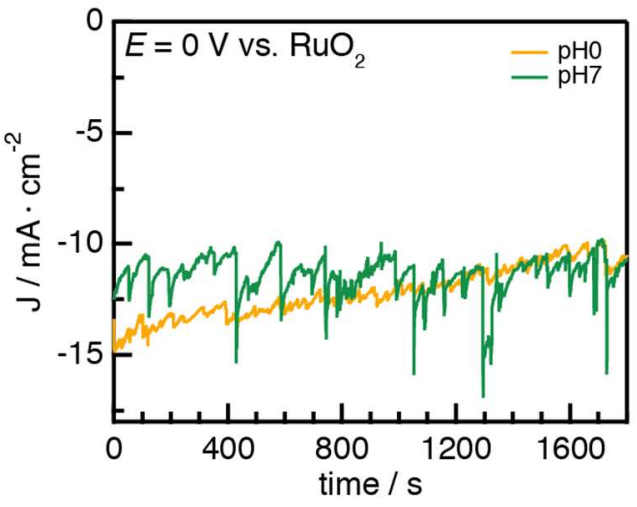

c

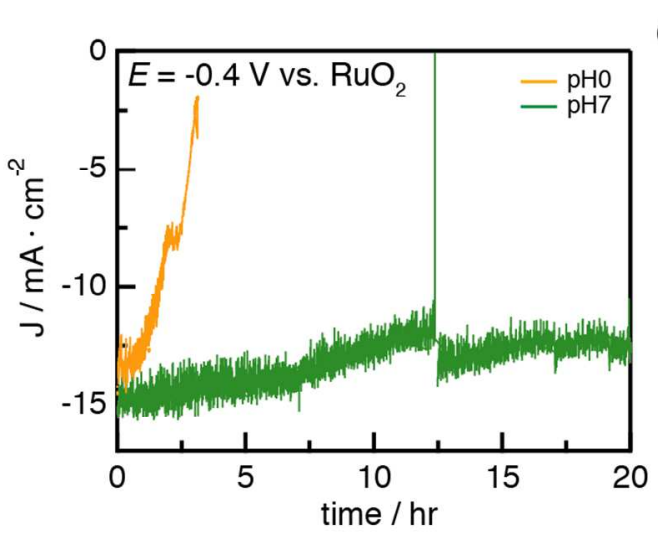

d

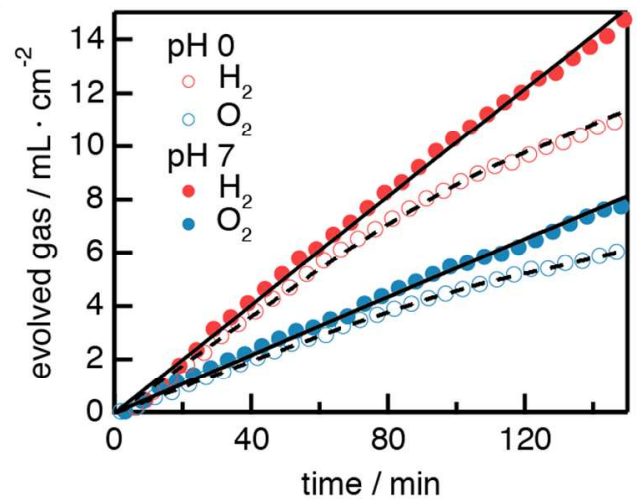


Figure 3. Output characteristics of the $\mathrm{RuO}_{2}-\mathrm{Ge} / \mathrm{GaIn} \mathrm{As} / \mathrm{GaInP} / \mathrm{AlInP}$-anatase $\mathrm{TiO}_{2}$ $\mathrm{Rh} /$ electrolyte dual junction tandem structure; (a) photocurrent-voltage characteristics in acidic ( $\mathrm{pH}$ 0), neutral ( $\mathrm{pH}$ 7) electrolyte and in neutral electrolyte including an AEM membrane; (b) chronoamperometric data of the initial temporal regime; (c) stability measurements at $-0.4 \mathrm{~V}$ vs. $\mathrm{RuO}_{2}$ counter electrode for acidic and neutral $\mathrm{pH}$; (d) hydrogen and oxygen gas collection for operation in acidic (open spheres) and neutral (full spheres) electrolyte. The measured gas volume for oxygen (blue symbols) and hydrogen (red symbols) is overlaid with the expected produced gas volume, as calculated from charge passed through the anode and cathode.

Fig. 3b gives the unassisted two-electrode photocurrent density vs. time for the initial operation regime, showing that while the photocurrent density decreases with time for acidic $\mathrm{pH}$, it remains more stable in neutral $\mathrm{pH}$ solutions. Chronoamperometric tests (at $-0.4 \mathrm{~V}$ vs. counter electrode as shown in Fig. 3c show that the device photocurrent density decreases in an acidic electrolyte to low values within $3 \mathrm{~h}$. However, in neutral $\mathrm{pH}$ electrolyte, stability over $20 \mathrm{~h}$ was demonstrated, with the photocurrent density remaining at $83 \%$ of its initial value (See comparative PEC test conditions and results in the Supplementary information section S8). In both cases, for $\mathrm{pH} 0$ and $\mathrm{pH}$, near unity Faradaic efficiency is confirmed through the agreement between the expected (solid line) and measured gas volumes (symbols) in Fig 3d. However, whereas the curves for $\mathrm{pH} 7$ stay linear with a constant gas production rate for $\mathrm{H}_{2} / \mathrm{O}_{2}$, as expected from the stability measurements, the curves for $\mathrm{pH} 0$ show a deviation from linearity due to the decreasing photocurrent. 
Etching of $\mathrm{TiO}_{2}$ is expected to occur at $\mathrm{pH} 0$ but not at $\mathrm{pH} 7$, as can be seen in the $\mathrm{TiO}_{2}$ Pourbaix diagram in Supplementary Section S10. Corrosion reactions can degrade the junction photovoltage, as well as lead to undercutting and removal of catalyst particles, thus reducing the exchange current of the Rh NP arrangement and a slowing of the HER kinetics. The system reacts also sensitively to series resistance changes, as illustrated by characteristics for devices employing an anion exchange membrane. The bend of the $\mathrm{J}-\mathrm{V}$ curve is shifted to cathodic potentials. However, device operation at pH 7 still yields a high STH efficiency of $18.5 \%$ and the device appears to be stable for a more extended period in accordance with predictions of $\mathrm{TiO}_{2}$ stability from thermodynamics. Even a slower reduction of the photocurrent is observed, we found that this photocurrent reduction could be partially reversed by emersion of the device from the electrolyte solution and applying a soft cleansing procedure (see in the Supplementary information section S8). The observation that the photocurrent can be partially restored appears to rule out loss of $\mathrm{Rh}$ catalyst particles, or even partial removal of the anatase interfacial layer, as causes of photocurrent reduction. We find, however, that the surface chemistry of Rh is influenced by the phosphate buffer of the neutral electrolyte: XPS in the Supplementary Section $\mathrm{S} 10$ clearly indicates $\mathrm{PO}_{4}$ formation on the Rh surfaces because of the absence of an In signal, which would have been observed in case of $\mathrm{InPO}_{\mathrm{x}}$ formation, concurrent with corrosion of the absorber. The photoelectrode regeneration procedure results in a $50 \%$ recovery of the photocurrent lost during the first $12 \mathrm{hrs}$ suggesting that the high porosity of the Rh NP layer inhibits full recovery by a short intermediate treatment. Employing a different electrolyte for pH 7 conditions might therefore benefit longterm-activity of the device. (See details in the Supplementary information section S10) 
Increasing the efficiency of a photoelectrosynthetic device from already high values towards theoretical limits is especially challenging. We have used a series of surface conditioning steps that have a twofold function: light management was drastically improved and the electronic properties were at least maintained. Compared to our earlier results ${ }^{5}$, we see an increase in the available cell voltage that is related to the increase in photocurrent at the counter electrode operation potential. Junction formation between the etched AlInP layer, $\mathrm{TiO}_{2}$ layer and Rh NPs suggest that the Fermi level alignment is nearly ideal.

Figure 4 shows a summary to date of selected STH efficiencies realized for monolithic integrated photoelectrosynthetic devices capable of unassisted water splitting.

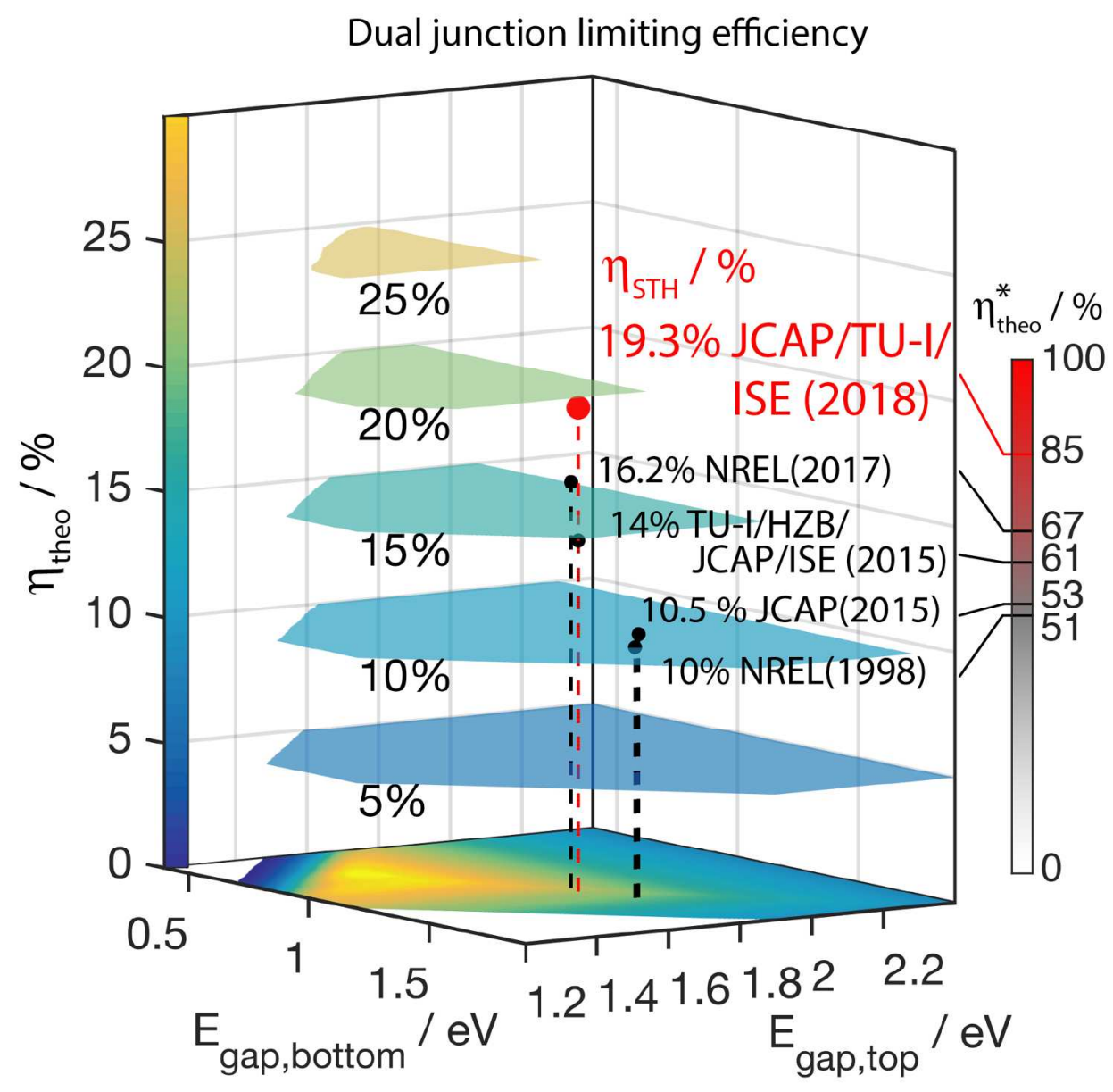


Figure 4. Comparison of realized limiting STH efficiencies and historic development. The analysis refers to a theoretical benchmarking value $\eta_{\text {theo }}$ (see text) and takes into account the top and bottom cell band gaps for the respective photolysis cells; also shown are the institutions of the contributing research teams. Abbreviations: NREL - National Renewable Energy Laboratory, USA; ISE - Institute for Solar Energy, Germany; JCAP - Joint Center for Artificial Photosynthesis, Caltech; TU-I - Ilmenau University of Technology, Germany; HZB - Helmholtz Zentrum Berlin, Germany. The bar chart on the right indicates the achieved efficiency with respect to the respective theoretical limit $\left(\eta_{\text {theo }}^{*}\right)$. See detail values in Supplementary Section S10.

Using the parameters shown in Table 1, our photoelectrosynthetic device reaches 0.85 of the theoretical limiting efficiency. It should be noted that the theoretical efficiency determined from the data in Table 1, is based on the best presently known electrocatalysts, a unity photoelectrode radiative efficiency and an absence of absorption losses ${ }^{9}$. We also calculated the STH efficiency as function of Tafel slope, exchange current density and ohmic drop to evaluate whether the optical or the electrochemical polarization losses dominate the solar cell performance. (See SI section S7, Fig. S17) Our record device is located in the region of highest efficiency showing that the optical loss is the limiting factor. However, the system, in principle, reacts sensitively to the polarization losses, emphasizing the importance of judiciously combining interface and catalyst.

Table 1: Approaches to theoretical limitation of light-induced photoelectrochemical water splitting; ideal, only exchange current density limited and devices that are optically and 
electrochemically limited are displayed in row 1-3, respectively. For the used band gap combination and only catalytic exchange current density $\left(\mathrm{J}_{\mathrm{XC}}\right)$ limitation, $\eta_{\text {theo }}=22.8 \%$ at AM $1.5 \mathrm{G}$ irradiation.

\begin{tabular}{|c|c|c|c|c|c|c|}
\hline & $\begin{array}{c}\mathrm{J}_{0, \text { cathode }} \\
{\left[\mathrm{mAcm}^{-2}\right]}\end{array}$ & $\begin{array}{c}\mathrm{J}_{0, \text { anode }} \\
{\left[\mathrm{mAcm}^{-2}\right]}\end{array}$ & $\mathrm{f}_{\mathrm{abs}}$ & $\mathrm{ERE}$ & $\mathrm{R}_{\mathrm{S}}[\Omega]$ & $\mathrm{R}_{\mathrm{Sh}}[\Omega]$ \\
\hline ideal & $\infty$ & $\infty$ & 1 & 1 & 0 & $\infty$ \\
\hline $\mathrm{J}_{\mathrm{XC}}$ limited & 1 & $10^{-3}$ & 1 & 1 & 0 & $\infty$ \\
\hline $\begin{array}{c}\mathrm{J}_{\mathrm{XC}} \text { and } \\
\text { optically } \\
\text { limited }\end{array}$ & 1 & $10^{-3}$ & 0.9 & 0.03 & 0 & $\infty$ \\
\hline
\end{tabular}

Stability appears to remain an issue of this photocathode device configuration, but we have demonstrated high efficiency in neutral electrolytes, and that extended operation of photocathode devices becomes possible if one can control the Rh surface chemistry. The use of Rh NPs with tailored size and shape distributions enables ultralow absorption. The future design of even more optimized tandem photoelectrodes appears to be possible, enabling solar fuel generation (water splitting, as well as $\mathrm{CO}_{2}$ or $\mathrm{N}_{2}$ reduction) efficiencies to be even higher than reported here, for example with STH champion device efficiencies of $>20 \%$ for integrated direct water photolysis being a realistic goal. 


\section{ASSOCIATED CONTENT}

Supporting Information. Supplementary Section S1-S11, Figure S1-S25, and Table S1.

Device fabrication; Materials characterization techniques; $\mathrm{TiO}_{2}$ characterization; Surface layer band alignment; Absorption enhancement by $\mathrm{TiO}_{2}$; Optimization of the optical design; Assessment of the solar-to-hydrogen efficiency measurement; Comparative PEC test conditions and results; Surface tension variation between $\mathrm{pH} 0$ and $\mathrm{pH} 7$; X-ray photoelectron spectra and mechanism development; and STH benchmarks.

\section{AUTHOR INFORMATION}

\section{Notes}

T.H., H.J.L, M.M.M., W.H.C., M.H.R. and H.A.A. conceived of the experimental study. W.H.C. and M.H.R. executed the experiments and did the data analysis. J.O., D.L. and F.D. prepared the tandem absorber. W.H.C., M.H.R., H.J.L., and H.A.A. wrote the paper and all authors commented on the manuscript. The authors declare no competing financial interests.

\section{ACKNOWLEDGMENT}

The authors acknowledge Katherine T. Fountaine for the calculation of theoretical photocurrent efficiencies of 2J PEC devices. This work was supported through the Office of Science of the U.S. Department of Energy (DOE) under award no. DE SC0004993 to the Joint 
Center for Artificial Photosynthesis, a DOE Energy Innovation Hub. Research was in part carried out at the Molecular Materials Research Center of the Beckman Institute of the California Institute of Technology. The work on tandem absorbers was funded by the German Federal Ministry of Education and research (BMBF) under the contract number FKZ 03F0432A (HyCon). M.M.M acknowledges funding from the fellowship programme of the German National Academy of Sciences Leopoldina, grant LPDS 2015-09.

\section{REFERENCES}

(1) Walter, M. G.; Warren, E. L.; McKone, J. R.; Boettcher, S. W.; Mi, Q.; Santori, E. A.; Lewis, N. S. Solar Water Splitting Cells. Chem. Rev. 2010, 110, 6446-6473.

(2) Xiang, C.; Weber, A. Z.; Ardo, S.; Berger, A.; Chen, Y.; Coridan, R.; Fountaine, K. T.; Haussener, S.; Hu, S.; Liu, R.; et al. Modeling, Simulation, and Implementation of Solar-Driven Water-Splitting Devices. Angew. Chem. Int. Edit. 2016, 55, 12974-12988.

(3) Fujishima, A. Electrochemical Photolysis of Water at a Semiconductor Electrode. Nature 1972, 238, 37-38.

(4) Khaselev, O.; Turner, J. A. A Monolithic Photovoltaic-Photoelectrochemical Device for Hydrogen Production via Water Splitting. Science 1998, 280, 425-427.

(5) May, M. M.; Lewerenz, H. J.; Lackner, D.; Dimroth, F.; Hannappel, T. Efficient Direct Solar-to-Hydrogen Conversion by in Situ Interface Transformation of a Tandem Structure. Nat. Commun. 2015, 6, 8286.

(6) Young, J. L.; Steiner, M. A.; Döscher, H.; France, R. M.; Turner, J. A.; Deutsch, T. G. Direct Solar-to-Hydrogen Conversion via Inverted Metamorphic Multi-Junction Semiconductor Architectures. Nature Energy 2017, 2, 17028.

(7) Lichterman, M. F.; Sun, K.; Hu, S.; Zhou, X.; McDowell, M. T.; Shaner, M. R.; Richter, M. H.; Crumlin, E. J.; Carim, A. I.; Saadi, F. H.; et al. Protection of Inorganic Semiconductors for Sustained, Efficient Photoelectrochemical Water Oxidation. Catal. Today 2016, 262, 11-23.

(8) Bae, D.; Pedersen, T.; Seger, B.; Iandolo, B.; Hansen, O.; Vesborg, P. C. K.; Chorkendorff, I. Carrier-Selective P- and N-Contacts for Efficient and Stable Photocatalytic Water Reduction. Catal. Today 2017, 290, 59-64.

(9) Fountaine, K. T.; Lewerenz, H. J.; Atwater, H. A. Efficiency Limits for Photoelectrochemical Water-Splitting. Nat. Commun. 2016, 7, 13706.

(10) May, M. M.; Lackner, D.; Ohlmann, J.; Dimroth, F.; van de Krol, R.; Hannappel, T.; Schwarzburg, K. On the Benchmarking of Multi-Junction Photoelectrochemical Fuel Generating Devices. Sustainable Energy Fuels 2017, 1, 492-503.

(11) Sathre, R.; Scown, C. D.; Morrow, W. R.; Stevens, J. C.; Sharp, I. D.; Ager, J. W., III; Walczak, K. A.; Houle, F. A.; Greenblatt, J. B. Life-Cycle Net Energy Assessment of 
Large-Scale Hydrogen Production via Photoelectrochemical Water Splitting. Energy Environ. Sci. 2014, 7, 3264-3278.

(12) Dimroth, F.; Beckert, R.; Meusel, M.; Schubert, U.; Bett, A. W. Metamorphic GayIn $\operatorname{In}_{1-}$ ${ }_{\mathrm{Y}} \mathrm{P} / \mathrm{Ga}_{1-\mathrm{X}} \mathrm{In}_{\mathrm{X}} \mathrm{As}$ Tandem Solar Cells for Space and for Terrestrial Concentrator Applications at C > 1000 Suns. Prog. Photovoltaics 2001, 9, 165-178.

(13) Ohlmann, J.; Sanchez, J. F. M.; Lackner, D.; Förster, P.; Steiner, M.; Fallisch, A.; Dimroth, F. Recent Development in Direct Generation of Hydrogen Using MultiJunction Solar Cells. AIP Conf. Proc. 2016, 1766, 080004.

(14) Porter, J. D.; Heller, A.; Aspnes, D. E. Experiment and Theory of "Transparent" Metal Films. Nature 1985, 313, 664-666.

(15) Degani, Y.; Sheng, T. T.; Heller, A.; Aspnes, D. E.; Studna, A. A.; Porter, J. D. "Transparent" Metals: Preparation and Characterization of Light-Transmitting Palladium, Rhodium, and Rhenium Films. J. Electroanal. Chem. 1987, 228, 167-178.

(16) Sanz, J. M.; Ortiz, D.; Alcaraz de la Osa, R.; Saiz, J. M.; González, F.; Brown, A. S.; Losurdo, M.; Everitt, H. O.; Moreno, F. UV Plasmonic Behavior of Various Metal Nanoparticles in the Near- and Far-Field Regimes: Geometry and Substrate Effects. $J$. Phys. Chem. C 2013, 117, 19606-19615.

(17) Anderson, R. L. Germanium-Gallium Arsenide Heterojunctions. IBM J. Res. \& Dev. 1960, 4, 283-287.

(18) Skorupska, K.; Pettenkofer, C.; Sadewasser, S.; Streicher, F.; Haiss, W.; Lewerenz, H. J. Electronic and Morphological Properties of the Electrochemically Prepared Step Bunched Silicon (111) Surface. phys. stat. sol. (b) 2010, 248, 361-369.

(19) Rizk, R.; de Mierry, P.; Ballutaud, D.; Aucouturier, M.; Mathiot, D. Hydrogen Diffusion and Passivation Processes in $P$ - And $N$-Type Crystalline Silicon. Phys. Rev. B 1991, 44, 6141-6151.

(20) Zhang, Y.; Pluchery, O.; Caillard, L.; Lamic-Humblot, A.-F.; Casale, S.; Chabal, Y. J.; Salmeron, M. B. Sensing the Charge State of Single Gold Nanoparticles via Work Function Measurements. Nano Lett. 2014, 15, 51-55. 